\title{
Envisioning, Evaluating and Co-Enacting Performance in Global Health Interventions
} Ethnographic Insights from Senegal

\author{
Diane Duclos, Sylvain L. Faye, Tidiane Ndoye \\ and Loveday Penn-Kekana
}

\begin{abstract}
Aвstract: The notion of performance has become dominant in health programming, whether being embodied through pay-for-performance schemes or through other incentive-based interventions. In this article, we seek to unpack the idea of performance and performing in a dialogical fashion between field-based evaluation findings and methodological considerations. We draw on episodes where methodological reflections on performing ethnography in the field of global health intersect with findings from the everyday practices of working under performance-based contracts in the Senegalese supply chain for family planning. While process evaluations can be used to understand contextual factors influencing the implementation of an intervention, we as anthropologists in and of contemporary global health have an imperative to explore and challenge categories of knowledge and practice. Making room for new spaces of possibilities to emerge means locating anthropology within qualitative global health research.
\end{abstract}

KeYWORDs: anthropology, ethnography, evaluation, global health, Senegal, supply chain

Historically, medical anthropologists have studied health and illness across places and cultures, combining a strong theoretical focus alongside an applied research orientation in collaboration with biomedical disciplines (Inhorn and Wentzell 2012). Anthropologists have also collaborated with development agencies for decades in various capacities, sometimes being expected to 'voice' the experiences of 'local communities' and sometimes determined 'to reveal more of the ethnographic detail of the organisational apparatus of development, as well as deeper analyses of the ways in which the concept of development has come to play a central role in our lives' (Lewis 2005). They have also immersed themselves in public service institutions, including hospitals and clinics, and health services in post-colonial settings (Jaffre and Olivier De Sardan 2003). Since the 1980s, critical medical anthropology has argued for the potential of ethnographies to not only reveal local belief systems about health and illness, but also to describe the role played by anthropologists 'to document and contextualize the effectiveness of health services as they impact people's lives' (Pfeiffer and Nichter 2008: 412). In the interdisciplinary field of global health, is qualitative research therefore another label used today to render visible what anthropologists have been doing all along? Or is anthropology being translated into something else within the development of qualitative enquiries of global health? Any attempt to 'locate' anthropology within the field of global health requires

Anthropology in Action, 26, no. 1 (Spring 2019): 21-30 (C) Berghahn Books and the Association for Anthropology in Action ISSN 0967-201X (Print) ISSN 1752-2285 (Online) doi:10.3167/aia.2019.260103

This article is distributed under the terms of the Creative Commons Attribution Noncommercial No Derivatives 4.0 International license (https://creativecommons.org/licenses/by-nc-nd/4.0/). For uses beyond those covered in the license contact Berghahn Books. 
addressing ways in which social imaginaries shaping health, illness and access to health services in various contexts are captured and interpreted. It also requires having conversations about the silos that exist between disciplines and about othering processes in health research. We argue that beyond exposing anthropology - or rendering it visible through its reconfigurations within the politics of global health - in order to make its role and added value accessible to a broader audience, the process of 'locating' is also an invitation for anthropologists to empower the representations of the world that they assemble in and out of these conversations.

In this article, the act of locating, understood as an approach to capturing epistemologies in the making and to reflecting on the role and responsibilities of anthropology as a community of practice, will be used to understand how 'performance' was both studied and co-enacted in the process evaluation of a supply chain intervention in Senegal. In other words, this article is not interested in describing qualitative methods as a range of research tools available to researchers, but in having a conversation about the methodological and theoretical implications at stake when translating qualitative research back into anthropology terms. We engage in this conversation by focusing on one core component of the intervention we were studying: the recruitment of third-party logisticians - referred to at our field site and thereafter in this article as private operators (POs) - by the nongovernmental organisation (NGO) implementing the intervention and working under performance-based contracting to deliver contraceptives and update inventories in health posts across Senegal. Under the supply chain model that we evaluated, contraceptive stock-out rates justified, in theory, the financial penalisation of contracted POs. Beyond presenting qualitative findings on a performance-based intervention, this article is interested in finding pathways to envision broader 'practical norms' that shape actors' action on the field without necessarily aligning with expected standards envisioned by funders and other relevant stakeholders (Olivier de Sardan 2008). Rodney Reynolds and Isabelle Lange (this issue) argue that anthropology ought to resist global imaginaries shaping global health and illness, and to propose alternative ways of seeing what is often obscured by an academic gaze oriented towards predefined issues. In today's global health moment, 'performing well' in a supply chain is tied up with being able to measure 'performance' through indicators. Along with Reynolds and Lange, we argue that such preconceptions about apprehending ways of 'performing well' might obscure essential social processes constituting the 'social lives' (Whyte et al. 2002) of supply chains.

We will first provide insight into the rationale and into some activities undertaken under the umbrella of the intervention that we evaluated in order to capture how the idea of a culture of performance was envisioned and approached by stakeholders - both as necessarily driven by private actors and also as measurable by indicators. We will also turn to the field of evaluation research to look at the role of anthropology in making the category of performance unstable and broaden its scope to include narratives of stock management. Second, we will account for specific episodes from the supply chain for family-planning commodities in Senegal in order to look at lived experiences of performance enactment and translations into team work transcending the dichotomy between public and private sectors. Third, we will reflect on ethnographic performances to account for different world-views emerging from global health evaluation research. Finally, we will argue that locating anthropology within an evaluation framework allows researchers to move beyond the successes or failures of a programme and make room for dynamic ontologies of performance to emerge in future global health realities.

\section{Envisioned Cultures of Performance}

In the early stages of our evaluation of this familyplanning supply chain intervention, we engaged with a certain 'culture of performance' narrative that was underpinning the programme's rationale. This narrative also shaped the intended function of the evaluation in assessing failures and successes, and it created an iterative space within which the role of anthropology was negotiated and performed.

\section{Background}

The London School of Hygiene and Tropical Medicine was appointed to conduct the evaluation of a supply chain model designed to prevent family-planning (FP) commodity stock-outs in health facilities in Senegal (Cavallaro et al. 2016). This new supply chain model was funded by a private philanthropic foundation and implemented in partnership with the Ministry of Health and Social Action in Senegal through an international NGO in order to address the issue of contraceptive stock-outs at service delivery points - even when contraceptives were available at the regional level (Daff et al. 2014). The funders of 
the evaluation, together with academic members of the broader interdisciplinary team, had expressed interest in dedicating time and resources to the qualitative component of the evaluation. The evaluation research activities spanned over a period of three years, from 2015 to the end of 2017, which allowed us to have an in-depth engagement with the intervention in the field. Our purpose was first to document and interpret how the intervention functioned in multiple contexts across Senegal. Ethical approval for this study was granted by the ethics committees of the Senegalese Ministry of Health and Social Action and the London School of Hygiene and Tropical Medicine. We conducted fieldwork across 11 regions of the country. Sampling was purposive to represent urban and rural areas and distance from familyplanning services. As you can see from our bios below, two of us were based at the London School of Hygiene and Tropical Medicine and the other two were based at Cheikh Anta Diop University in Dakar. We also recruited four research assistants from the Department of Sociology at Cheikh Anta Diop University as part of our team.

The methods that we used included reviewing funding documents, proposals and project reports related to the supply chain intervention. We also conducted in-depth interviews with key national and international stakeholders; in-depth interviews with all cadres of personnel involved in the supply chain and family-planning activities at the regional, district and facility levels (including clinic staff, programme auditors and POs) in order to examine views on performance-based contracting, understand how the intervention was modified in different contexts, and learn about potential unanticipated issues. We asked nurses in charge of clinics to fill in fortnightly reflective diaries on issues relating to the implementation. We also observed coordination meetings at the district level of the health system. In addition, we carried out ethnographic work to understand the logics and practices of implementation on the ground: researchers travelled with POs while performing deliveries and carrying out stock inventories, and they observed the lived realities of POs, facility stockists and providers at work. This presented a further opportunity to discuss collectively their perspectives on the project. Ethnographic studies have helped researchers understand contexts in which performancebased contracting was the operational framework, and the knowledge generated from these studies has also been used to challenge neoliberal understandings of health system interventions (Magrath and Nichter 2012). Along this trend, the data presented below sheds some light on the social life of a supply chain and the unstable character of performance-in-the-making.

\section{Performing Well through Stock Availability}

To address high rates of contraceptive stock-outs in health facilities in Senegal, the intervention sought to introduce a 'culture of performance' borrowed from the private sector, which assumed that performance could be measured quantitatively by stock availability and timeliness of deliveries. One of the core components of the supply chain model that our research team evaluated was the introduction of private businesses deploying POs working under pay-for-performance contracts to update stocks in facilities, collect contraceptives from regional storerooms and deliver them to health facilities in order to ensure contraceptive availability and free up time for health workers to do other tasks. Under the former family-planning supply chain model in Senegal, public facilities were required to order contraceptives from and submit stock requisitions for family-planning commodities to district personnel. After the orders were filled, each facility was in charge of collecting their commodities from district storerooms. In Senegal, a stockist is contracted by the local health committees to work in each facility storeroom. With the intervention under evaluation, the new POs introduced were recruited and supervised by the implementing international NGO to undertake monthly inventory and 'top-up' stock as necessary. Payments to POs were based on their performance, with penalties if stock-outs were to rise above two per cent. Activities related to this intervention were coordinated by assistant logisticians operating at the district level and pharmacists seated at the regional level. A monitoring and evaluation team was also dedicated to the project at the national level. Under this supply chain model, 'a contraceptive stock-out was defined as zero units available for sale at the facility on a day when the facility was open' (Daff et al. 2014). POs refilled stocks to a level calculated based on a three-month consumption level (Cavallaro et al. 2016). The rationale behind this intervention suggests that stock-outs were demand experiences at point of sale represented through numbers. However, by reproducing the definition of stock adopted as a management tool in this intervention - that is, by capturing a stock-out predominantly through the recording of numbers researchers ran the risk of masking the complexities and local embodiments of stock management in supply chains. We asked ourselves: How does contraceptive stock management translate into practice? Does this idea of 'performance' envisioned in the design 
of the intervention encounter other imaginaries and practices? And if so, which ones?

While reviewing the study protocol and policy documents related to the intervention and familiarising ourselves with the intervention's theory of change, the language around the performance of private actors innovating in contrast with a static and inefficient supply chain model came across rather clearly:

I think our intervention brought visibility on delivery performance, so this is a great tool for understanding where there are facilities with high performance and where there are weak performances, and to start problem-solving and learning. It enabled a culture of performance management that did not exist in Senegal before, and that's an enormous success. (Interview with a funder)

Unpacking this idea of a 'culture of performance' is key not only to understanding expectations associated with bringing the private sector into the health supply chain, but also to understanding how this idea intersects with or disconnects from health providers' understandings and practices in health facilities.

\section{Global Health Evaluation Research and Performance Metrics}

In a book on audit cultures, Marilyn Strathern (2000) has highlighted the new regimes of accountability and efficiency governing workplaces, institutions and individuals. While public health projects' evaluations are part of this audit culture, they also increasingly offer room to understand complex contexts of implementation (Byrne 2013) and therefore provide opportunities for anthropologists to collect rich ethnographic material that can contribute to the critique of such regimes. Yet, in an evaluation framework constrained by programmatic imperatives and predefined reporting mechanisms, to what extent can we create spaces where social and cultural translations of health interventions can be captured?

Throughout this article, we acknowledge important existing debates surrounding organisational cultures of performance (Scott et al. 2003) in order to revisit performance episodes emerging from this supply chain evaluation as a means to reflect on imagined and practised qualitative enquiries. Vincanne Adams and colleagues (2014) invite us to think about alternative approaches in a global health field governed by innovation, driven by statistically based research and fascinated by technological fixes. In the era of the millennium and sustainable development goals targets, and of public-private partnerships, the notion of performance has become dominant in health programming and is reinforced through pay-forperformance interventions and other incentive-based programming. Performance has been framed within the broader trend 'often termed "neoliberal", which assumes markets to be the most effective mechanism for allocating resources and achieving results in the public as well as private sectors' (Magrath and Nichter 2012: 1779). Performance-based schemes implemented in low- and middle-income countries have more recently been studied through qualitative research, which has unravelled the contexts of implementation as well as the long-term effects of these programmes (De Allegri et al. 2018).

According to João Biehl, the new goal of evaluation research to identify programmes that work and that can be scaled up or replicated 'is displacing the previous goals of interventions, making the provision of actual health services secondary to the development of reliable methodologies and the generation of comparable data. Metrics are presented as objective, value-free, and abstracted from social and political contexts' (2016: 129). Fostering performance is at the core of the supply chain model that we evaluated. In evaluation research, anthropologists also perform ethnography in certain ways when negotiating epistemologies with other disciplines. John Law and John Urry (2004) have demonstrated that social sciences are simultaneously embedded in and productive of social realities. While the use of social sciences to inform process evaluations is primarily seen as influencing practice by identifying how interventions that work can be scaled up or implemented in other contexts, realities are also produced by the co-enactment of performance across and beyond ethnographic encounters.

\section{Performance Episodes from the Family- Planning Supply Chain in Senegal}

The recent programmatic and research focus on supply chains in health systems sheds light on social and cultural dynamics shaping the stocking, inventorying and procurements of commodities beyond quantitative categories in different settings (Muyinda and Mugisha 2015). The health supply chain in Senegal is driven by a donor-oriented approach, where each programme operates within its own distribution system. Understanding a supply chain intervention entails engaging with its language and its embodiment in practices, relationships, institutions and poli- 
cies, as well as with its constant reconfigurations and negotiations in space and time.

As mentioned above, the intervention introduced POs as outsiders in order to trigger a 'culture of performance' in the health system. The words 'efficiency' and 'creativity' were associated with the private character of the enterprise:

If we delegate work to a private [actor], even if it has a cost, at least we know it is efficient. There is efficiency. (Interview with a Senegalese government member)

However, POs' definition of performance on the ground as well as their aspirations appeared to be broader than the liberal understanding at the core of the performance-based contract scheme designed by national and international stakeholders:

The job is good, thank God. We do it to fight the dangers (unwanted pregnancies) faced by certain persons, and we enjoy it. That is why the work is neither easy nor difficult. We enjoy it because we cannot leave people facing danger if we can do something about it; this is why we do it with an open heart, and we are very committed. (Interview with a PO)

Some POs showed interest in contributing to the health system in order to improve women's health, implying that they were not only driven by achieving the targets inscribed in their contract. POs felt that they were a part of the project beyond the logistics and sometimes took pride in contributing to promoting women's health. One of the entrepreneurs managing POs working for the intervention acknowledged officially being part of the project as a 'partner' with the implementing NGO, but also admitted to somehow feeling reduced to the position of logistician or employee:

I would like things to go forward towards a real partnership that will be mutually beneficial because we need to protect this project. Well, we need to accompany this project because I am going to tell you something. In M., we had problems with village chiefs. Sometimes they come to us in the health posts and tell us they don't agree regarding ... It happened to me four or five times (It is true that I have never reported on this because as far as I understand I am here to deliver the commodities). But they came to tell us they don't agree with family planning and Islam forbid that. We need solutions not to stop births (because they think it is trying to be stopped) but to help birth spacing. All the things we hear, all the things we see: they can help the global strategy. (Interview with a PO)
This PO was keen to get more involved in what he called the 'global strategy' of the project and expressed frustration about being constrained by his contractual obligations and the related reports that he and his team had to produce. According to him, there was no space where POs could use their diverse sets of skills to affect and improve the development of the project. His words convey a gap in the performances as defined in figures and as defined in his vision of being able to thrive in his professional position.

Stakeholders' interviews and official presentations of the intervention did not expand on the strategy that was used to introduce POs into the public health system. In order to succeed in its mission to deliver enough contraceptives to avoid stock-outs on a regular schedule, POs had to prove that they understood social norms governing health facilities. During observations that we conducted with POs, a recurrent pattern emerged: on arrival at a health facility, the $\mathrm{PO}$ would greet the clinic manager and then head to the pharmacy to update the stockist. When we discussed this routine with the PO, they explained how the assistant logisticians supervising them had advised them to respect the hierarchy of the health system and always meet the managers when delivering. Interestingly, the supervisors in question were mainly former district or regional reproductive health supervisors. As former members of the Senegalese health system, these professionals were able to explain the codes to the 'outsiders' and facilitate access to health posts. At the core of the inventory process was the relationship between the stockist managing the storeroom at the facility level and the PO:

Once, when I was on another route, he [the stockist] refused to let the other team in. The other operator phoned me, and I called the stockist. He didn't pick up the phone. ... I called the district chief doctor, and after he intervened the stockist accepted letting the team in. (Interview with a PO)

Stockists were key interlocutors for POs, for they allowed them to access storerooms and conduct inventories. POs relied on the consumption data from previous months to forecast the number of contraceptives that they would need for the current month. However, family-planning outreach activities were sometimes planned by health posts. During these activities, higher numbers of contraceptives were distributed to the community. Observations showed that some midwives approached POs during the inventory count to inform them about upcoming activities 
and to request more supplies. Nurses and midwives were therefore important partners from whom POs could gain important consumption forecast information. Adjustments, negotiations and collaborations characterised this relationship. This example shows that the calculation of stocks could not rely exclusively on consumption data but also required detailed knowledge of a health post's activities. For the 'outsider', it required more than stock-management skills.

Furthermore, over the course of our fieldwork we observed coping strategies that were developed by private and public actors that prevented stock-outs from being recorded:

Yes, all the products (types of contraceptives) are available now. Once I had clients asking for a product. We had only three boxes left. Madame X asks us not to use those boxes to avoid stock-outs. (Interview with a stockist)

Our informants emphasised the fact that the POs, aware of the situation, would ideally come to the post and top up products, meaning that in effect there is a stock out that cannot be captured by performance metrics. This strategy might look anecdotal at the intervention level, but it nevertheless illustrates the difficulties associated with understanding a stock-out exclusively in numeric terms. Products can be visible on the shelves in a health post's storeroom, but yet not made available to clients in order to satisfy narrowly defined performance incentives. Another illustration of the need to go beyond the stock-out rate to capture access to contraceptives for clients at the time of the evaluation was the fact the supply of auxiliary products that were needed to administer some of the contraceptives (such as syringes, xylocaine and cotton wool) were not integrated into the intervention, or the fact that the autoclaves used to sterilise material often did not work. These situations meant that while women's preferred method of contraception might be in stock, family-planning providers still did not have the material and equipment needed to offer that method.

Being able to access storerooms in health facilities is a pre-requisite for POs to undertake the inventorying activities they are contracted for:

I: So, if I understand what you are saying, when you can't deliver in some posts, you wait for the following month?

PO: We consider them remaining health posts. It depends. If for instance they are not there when we deliver, we wait for the following month. If we arrive and people are present but busy, we do our best to see them and work with them. It is not like you visit the post and you don't find anyone. Sometimes, when we arrive, the health post is closed. We call them, and they can't come. If this is the case, we come back in the following month. (Interview with a private operator)

Access to storerooms for operators seems to be embedded in social relationships that evolve over time:

Sometimes the operator comes, and I am not informed. Sometimes I come into the pharmacy and find him. I tease him sometimes, asking him why he does not come and greet me; I am the one selling his products! (Interview with a midwife)

Interestingly, the day-to-day management of performance-based contracting by the NGO that implemented the intervention translated into a set of supervision activities where POs were getting support at different stages of their work. The relationship between POs, assistant logisticians supporting them at the district level, zone pharmacists and the implementing NGO monitoring and evaluation team at the central level constituted a complex network, in which a vast quantity of information was circulated. The fact that former members of the health system were amongst the assistant logisticians and pharmacists added a layer of complexity and somehow broke the verticality of the supply chain. Within this network, we tried to understand the mechanisms that could lead to the penalisation of a PO. Mentioning the penalisation component of the intervention triggered discomfort amongst the intervention-implementing NGO staff members. Assistant logisticians and pharmacists defended themselves against being in charge of auditing the POs and in contrast valued their roles as supervisors and mentors. During the time that our evaluation team spent in the field, no record of penalisation was found. What we found was a team composed of members who were dedicated to reaching the objectives of the project together. This way of translating auditing into collaboration questions, in our view, the social imaginaries surrounding the idea of performance, which is sometimes reduced to being triggered by competition. Our data sheds light on a collective interpretation of performance through aligned interests in avoiding contraceptive stockouts. Furthermore, through these collaborations between POs and health providers, the expectations and practices that made up distinctions between health system 'insiders' and third-party 'outsiders' were more alike than different - rendering the distinctions rather blurry. 


\section{Performing Ethnography in and out of Interdisciplinary Conversations}

In this section, we will twist the concept of performance from researching performance-based contracting to exploring anthropologists' own ethnographic performances in order to highlight the power of anthropology to co-produce realities (Law and Urry 2004) and the need to account for these enactments in global health thinking. We simply understand ethnographic performances to be how anthropologists stage their research. When accounting for representations and enactments of performance, anthropologists necessarily engage with conflicting ontologies. In the case study we presented here, it involved the public and private sectors and associated ideas of efficiency, flexibility, change and immobilism. Addressing and potentially challenging those ontologies within the implementing frame of an evaluation setting, and within an interdisciplinary academic environment, shape the ways in which ethnography is performed and sometimes translated into qualitative research. The hazy label of 'qualitative research' extensively used in global health research can trigger both comforts and discomforts for anthropologists involved therein. On the one hand, doing qualitative research facilitates collaborations between sociologists, anthropologists, historians, and geographers (and so on) to undertake research on objects of common interest (such as family-planning use). On the other hand, in a field driven by innovative programmatic approaches, qualitative research has been used as a quick way to 'fill the gaps' (Reynolds 2016) and understand what contextually facilitates or hinders health interventions, often at the expense of our understanding of how individuals and communities relate to the world in specific settings. How can we touch upon local knowledge - that is, the everyday categories composing the social worlds of our informants - in a multi-disciplinary evaluation?

Let us now turn back to the episode described above, the one where a stockist decided not to sell contraceptives to a woman seeking family-planning services in her facility, in order to avoid recording a stock-out in the facility. This account can enlighten or obscure different key social processes according to the research and implementation context in which one chooses to locate it. If one locates this account in an evaluation research paradigm where success is measured by the compliance between what is found on the ground with what was anticipated could work in a protocol, not selling a contraceptive to a woman can easily be interpreted as a failure. If one locates this episode in a health system context where a woman is denied access to a contraceptive, this can be interpreted as a deviance. However, if one locates this episode in an intervention context where stock-out rates are at the centre of attention, on the one hand, and where performance seems to be enacted within a collective supervised frame as opposed to an individual audited one, on the other hand, such episode produces knowledge on rational social conduct that can be acknowledged to develop locally grounded categories of practice. In this sense, although anthropologists' role as data collectors qualitatively assessing an intervention along with other research designs (such as epidemiological and costing approaches) is crucial to the understanding of complex interventions, this role is not sufficient. Anthropologists are also well positioned for stepping back from pre-established definitions and measurements of success to value and empower stories emerging when attempting to drive organisational change. Such stories may emerge through different pathways, including through negotiating methodologies and scales across disciplines.

\section{Opening-Up Spaces of Possibilities?}

In context, we showed that a stock-out cannot be identified solely by the absence of a commodity on the shelf. The relational dimension of stocks bringing together actors from the public and private sectors requires an epistemological rupture with the idea of supply chains as vertical structures (in our case study, one going from the national level to sub-national level). Furthermore, we showed how anthropology can help us reflect on the drive for performance understood in technical terms (such as stock-out rates) by reading efficiency narratives in the context of day-to-day social practices (embedded in institutions and relationships). The episodes we presented can in turn challenge contractual definitions of performing. Finally, acknowledging the centrality of stock availability for women's access to the contraceptive of their choice when they go to a health facility, we also explored how, when it attempts to compete for 'evidence legitimacy' with metrics developed in other disciplines, anthropology can be caught up in a performing paradigm using stories to validate or invalidate pre-conceived solutions. In contrast, we believe that anthropology can 'open the windows on other possible forms of human social existence; because it serves as a reminder that what we assume to be immutable has been, in other times and places, 
arranged quite differently, and therefore that human possibilities are in almost every way greater than we ordinarily imagine' (Graeber 2007: 1).

Ethnographic work was essential to capturing how POs performed in a specific social and cultural context. In our case study, the POs were not neutral outsiders but performance-based contractors who were social agents translating the intervention into local settings. Conducting ethnographic work in the supply chain as we did was to question the role of anthropology in evaluation settings by confronting the category of 'performance' as imagined by the funders and implementers of the intervention and applied by them to practices and discourses performed routinely on the ground. Including everyday practices and translations of performances in the context(s) and relationships that shaped them required moving from a 'successes versus failures' understanding of an intervention to a thorough documentation of dynamic processes and routines. Ethnographic approaches to performance therefore question the role of qualitative evidence in global health research and the use that can be made of quantitative evidence in relation to complex implementation contexts. Where do the human narratives emerging from a supply chain study stand in the broader evaluation, and how can they be empowered as evidence? How is the ethnography seen and validated (or not) within the project and the wider community? How can such findings be used by funders and implementers?

The entanglement of repertories and practices emerging from ethnographic encounters constitutes a dynamic frame in which performance is enacted. Law and Urry (2004) have emphasised the power of social enquiries to enact the social. In evaluation research, engaging with these enactments requires making the border between what constitutes or does not constitute the evaluated intervention unstable and to account for world-views emerging from ethnographic encounters. For the PO, her or his ability to update stocks and deliver products on time depended also on her or his ability to integrate into the health system and to collaborate with the stockists in the storerooms. Facilitating the POs' work was also their ability to engage with providers of familyplanning services - midwives in particular - as a way to gain information on possible outreach activities that would increase the distribution of contraceptives and put their facilities at risk of experiencing a stockout. Such lived experiences of enacting performance are not mere illustrations of an intervention subjected to adjustments being implemented in different contexts. They offer insight into ways of belonging to health systems. Ethnography offers tools which we can use to step back from qualitatively assessing the logistical structure of the supply chain for family planning as a corridor where different levels of the health system (national, regional, district, local) are related to each other to make room for broader modalities of collaboration and ethos to emerge. The two per-cent stock-out rate theoretically defining the threshold for penalisation under the contracts signed by third-party operators obscure the realities of the implementation of the supply chain intervention if no attention is brought to the complex processes leading to that number. Drawing on ethnographic data, it appears that contraceptive stock-out rates did not directly account for POs' logistical and technical performance, but rather accounted for collective achievements and negotiations between public and private actors that unfolded over time in the supply chain. During a dissemination workshop with the NGO that implemented the intervention, it was confirmed that the model had moved away from a penalisation system where POs were audited, to a supervision model where formative measures were taken to prevent POs from failing. In the mindset of a qualitative process evaluation, this can be interpreted as performance-based contracting not being considered as an acceptable tool in Senegal to address stock-out issues. However, by assembling those representations of performance, anthropology can make the category of performance relevant as an operational tool through which collaborations, ideals, commitments and ambitions can be integrated into global health thinking.

Evaluation criteria established by donors in the evidence-based movement influenced the field of global health by associating the effectiveness of health interventions with quantitatively defined results. An ethnographic evaluation design, by situating health interventions in the lives of systems, communities and individuals, can critically inform the 'anti-politics' of international development which have increasingly promoted 'technical solutions to technical problems' (Ferguson 1994) and, more particularly, can challenge 'the stage of evidence and evaluation' (Birn 2009) that we are experiencing in global health by highlighting harder-to-measure but yet critical features of interventions. Translating qualitative research back to more anthropologically situated knowledge in interdisciplinary evaluation contexts creates room for deconstructing a certain ontology of performance, and its multiple enactments in different contexts need to be further explored. Challenging a narrowly focused definition of per- 
formance resonates with broader conversations on channelling various forms of knowledge production as legitimate evidence to imagine spaces of possibility in global health research and policy. The empowerment of stories from the supply chain should not be seen as a way to compete with or complement quantitative metrics of performance, as this could in the end reinforce pre-existing categories and thus reflect more of an audit-oriented global health landscape (Storeng and Béhague 2014). Finding the right distance from which to examine and account for stories in global health is a necessary step towards challenging categories that become taken for granted at the expense of dynamic local realities. In this sense, locating anthropology within qualitative global health research is also an opportunity for anthropologists as a community of practice to enact the social through constantly grounding categories in plural worlds (Law and Urry 2004).

\section{Conclusions}

Beyond statistical data defining stock-outs and performances, supply chains are social and political spaces that shape the outcomes of health interventions but that need to be understood beyond the scope of interventions. The ethnographic episodes described in this article informed discussions on local implementations of an intervention by making the borders of what defines performance unstable. Examining numbers only to assess performance can reinforce prescriptive logics of global health programmes, such as the supposed verticality of the supply chain. In contrast, looking at narratives and numbers in conversation triggers a grounded understanding of how global health interventions are inhabited and reclaimed locally. In this study, it appears that developing partnerships with health system actors such as stockists, midwives and nurses in charge of clinics was essential for accessing storerooms and relevant information to top up contraceptive stocks in health facilities. Furthermore, it appeared that stock (un) availability cannot be understood outside the lived experiences and narratives constituting them. Empowering actors' experiences and narratives as evidence in global health research is key to (re)defining what success is and how it gets measured (or not). While process evaluations can be used to understand contextual factors influencing the implementation of an intervention, ethnographic data emerging from qualitative evaluation research can also be assembled to reflect on norms and practices affecting how peo- ple live and work together. Locating anthropology within qualitative global health research is therefore also about taking responsibility as anthropologists in and of contemporary global health to explore and challenge categories of knowledge and practices, while acknowledging our own performative roles as global health players, and to open up spaces for possibilities that can shape the logistics of framing and implementing locally grounded health programmes.

\section{Acknowledgements}

This research is supported by funding from MSD through its MSD for Mothers programme. MSD had no role in the design, collection, analysis, and interpretation of the data or in the writing of this article, let alone the decision to submit this article for publication. The content of this article is solely our responsibility and does not represent the official views of MSD. MSD for Mothers is an initiative of Merck \& Co., Inc., which is headquartered in Kenilworth, New Jersey.

All four of us are extremely grateful to all the participants who dedicated time to this research. We also wish to thank the whole study team, and in particular Mareme Diallo, Finou Therese Mendy, Sega Gueye and Khady Diallo. We are also grateful to the anonymous reviewers and to the editor of Anthropology in Action, Christine McCourt, whose thoughtful comments helped improve this manuscript. Finally, we are grateful to Isabelle Lange and Rodney Reynolds for initiating this conversation with us and for their intellectual support and patience during the process of putting this special issue together.

Diane Duclos is an anthropologist based at the London School of Hygiene and Tropical Medicine (LSHTM). Before joining the LSHTM, Diane completed a PhD in the anthropology of development at the Graduate Institute of International and Development Studies, Geneva.

Email: diane.duclos@lshtm.ac.uk

Sylvain L. FaYe is a socio-anthropologist based at the Department of Sociology at Cheikh Anta Diop University in Dakar with extensive experience in the field of global health. Sylvain holds a PhD from the University of Bordeaux, France.

Email: sylvain.faye@ucad.edu.sn

Tidiane Ndoye is a socio-anthropologist with expertise in global health. He is the head of the Depart- 
ment of Sociology at Cheikh Anta Diop University in Dakar. Tidiane holds a PhD from the School for Advanced Studies in the Social Sciences in Marseille, France. Email: tidiane.ndoye@ucad.edu.sn

Loveday Penn-KeKana is a medical anthropologist at the London School of Hygiene and Tropical Medicine (LSHTM) with 20 years of experience working as an activist, programme manager, and technical advisor in the South African Department of Health and as an academic in the field of maternal health and health systems with a particular interest in the health workforce. Email: loveday.penn-kekana@lshtm.ac.uk

\section{References}

Adams, V., N. J. Burke and I. Whitmarsh (2014), 'Slow Research: Thoughts for a Movement in Global Health', Medical Anthropology 33, no. 3: 179-197. doi:10.1080/01459740.2013.858335.

Biehl, J. (2016), 'Theorizing Global Health', Medicine Anthropology Theory 3, no. 2: 127-142. doi:10.17157/ mat.3.2.434.

Birn, A. E. (2009), 'The Stages of International (Global) Health: Histories of Success or Successes of History?' Global Public Health 4, no. 1: 50-68. doi:10.1080/17441690802017797.

Byrne, D. (2013), 'Evaluating Complex Social Interventions in a Complex World', Evaluation 19, no. 3: 217-228. doi:10.1177/1356389013495617.

Cavallaro, F. L., D. Duclos, R. F. Baggaley, L. PennKekana, C. Goodman, A. Vahanian, A. C. Santos, J. Bradley, L. Paintain, J. Gallien, A. Gasparrini, L. Hasselback and C. A. Lynch (2016), 'Taking Stock: Protocol for Evaluating a Family Planning Supply Chain Intervention in Senegal', Reproductive Health 13, no. 45. doi:10.1186/s12978-016-0163-7.

Daff, B. M., C. Seck, H. Belkhayat and P. Sutton (2014), 'Informed Push Distribution of Contraceptives in Senegal Reduces Stockouts and Improves Quality of Family Planning Services', Global Health: Science and Practice 2, no. 2: 245-252. doi:10.9745/ GHSP-D-13-00171.

De Allegri, M., M. P. Bertone, S. McMahon, I. M. Chare and P. J. Robyn (2018), ‘Unravelling PBF effects beyond Impact Evaluation: Results from a Qualitative Study in Cameroon', BMJ Global Health 3, no. 2. doi:10.1136/bmjgh-2017-000693.

Ferguson, J. (1994), The Anti-Politics Machine: Development, Depolitization and Bureaucratic Power in Lesotho (Minneapolis: University of Minnesota Press).
Graeber, D. (2007), Possibilities: Essays on Hierarchies, Rebellion, and Desire (Edinburgh: AK Press).

Inhorn, M. C. and E. A. Wentzell (eds.) (2012), Medical Anthropology at the Intersections: Histories, Activisms, and Futures (Durham, NC: Duke University Press).

Jaffre, Y. and J. P. Olivier De Sardan (eds.) (2003), Une médecine inhospitalière: Les difficiles relations entre soignants et soignés dans cinq capitales d'Afrique de l'Ouest (Paris: Karthala).

Law, J. and J. Urry (2004), 'Enacting the Social', Economy and Society 33, no. 3: 390-410. doi:10.1080/03085 14042000225716.

Lewis, D. (2005), 'Anthropology and Development: The Uneasy Relationship', in J. G. Carrier (ed.), A Handbook of Economic Anthropology (Cheltenham, UK: Edward Elgar), 472-486. Available online at http:// eprints.lse.ac.uk/253/.

Magrath, P. and M. Nichter (2012), 'Paying for Performance and the Social Relations of Health Care Provision: An Anthropological Perspective', Social Science \& Medicine 75, no. 10: 1778-1785. doi:10.1016/j.socscimed.2012.07.025.

Muyinda, H. and J. Mugisha (2015), 'Stock-Outs, Uncertainty and Improvisation in Access to Healthcare in War-Torn Northern Uganda', Social Science E Medicine 146: 316-323. doi:10.1016/ j.socscimed.2015.10.022.

Olivier De Sardan, J. P. (2008), 'Researching the Practical Norms of Real Governance in Africa', Africa Power and Politics Research Paper 5 (London: ODI).

Pfeiffer, J. and M. Nichter (2008), 'What Can Critical Medical Anthropology Contribute to Global Health?' Medical Anthropology Quarterly 22, no. 4: 410-415. doi:10.1111/j.1548-1387.2008.00041.x.

Reynolds, J. (2016), '“Missing Out": Reflections on the Positioning of Ethnographic Research within an Evaluative Framing', Ethnography 18, no. 3: 345-365. doi:10.1177/1466138116664106.

Scott, T., R. Mannion, H. Davies and M. Marshall (2003), Healthcare Performance and Organisational Culture (London: CRC Press).

Storeng, K. T. and D. P. Béhague (2014), '“Playing the Numbers Game": Evidence-Based Advocacy and the Technocratic Narrowing of the Safe Motherhood Initiative', Medical Anthropology Quarterly 28, no. 2: 260-279. doi:10.1111/maq.12072.

Strathern, M. (ed.) (2000), Audit Cultures: Anthropological Studies in Accountability, Ethics and the Academy (London: Routledge).

Whyte, R. S., S. Van der Geest and A. Hardon (2002), Social Lives of Medicines (Cambridge: Cambridge University Press). 